Revue d'histoire de l'Amérique française

REVUE D.HISTOIRE DE L'AMÉRIQUE FRANÇAISE

\title{
La première compagnie de Miscou, 1635-1645
}

\section{Robert Le Blant}

Volume 17, numéro 3, décembre 1963

URI : https://id.erudit.org/iderudit/302289ar

DOI : https://doi.org/10.7202/302289ar

Aller au sommaire du numéro

Éditeur(s)

Institut d'histoire de l'Amérique française

ISSN

0035-2357 (imprimé)

1492-1383 (numérique)

Découvrir la revue

Citer cet article

Le Blant, R. (1963). La première compagnie de Miscou, 1635-1645. Revue

d'histoire de l'Amérique française, 17(3), 363-370.

https://doi.org/10.7202/302289ar d'utilisation que vous pouvez consulter en ligne.

https://apropos.erudit.org/fr/usagers/politique-dutilisation/ 


\section{LA PREMIËRE COMPAGNIE DE MISCOU 1635-1645}

Le territoire de la concession accordée, le 15 novembre 1632 , par la Cie de la Nouvelle-France à une Cie particulière pour la traite des pelleteries dans le Saint-Laurent ne commençait qu'à partir de Miscou ${ }^{1}$. On ne connaît pas la date de la concession de cette région à une première Cie particulière dont la formation est antérieure au 5 janvier 1636. Les associés donnèrent alors la charge de recevoir leurs cotisations ${ }^{2}$ à Jacques Berruyer, sieur de Manselmont, dont l'identité est précisée par des actes postérieurs. Membre de la Cie des Cent-Associés, figurant au No 16 de la liste rectifiée par nous, frère de Georges Berruyer, sieur de Bernesault ( $\left.\mathrm{N}^{\circ} 123\right)$, appartenant à une famille tourangelle ${ }^{3}$, il encaissa, le 5 février 1636, de François Fouquet $\left(N^{\circ} 140\right)$, père du futur surintendant des Finances, 2,000 livres pour deux des trente-deux parts de la société ${ }^{4}$. Celle-ci était vraisemblablement organisée sur un mode inspiré de celui de la Cie de la Nelle-France où l'on trouve des préposés pour des affaires particulières qui ne doivent pas être confondus avec les directeurs des Cent-Associés. L'habitation de la baie de Miscou était ainsi, le 12 avril 1636, entre les mains de Jacques Berruyer, Antoine Cheffault ( $\mathrm{N}^{\circ} 62$ ) et Jean Rozée ( $\left.\mathrm{N}^{\circ} 36\right)$, le fameux marchand de Rouen. Ils étaient préposés par la Cie de la Nouvelle-France aux embarquements pour cette destination, formule qui semble indiquer au moins pour cette époque une collaboration des Cent-Associés dont tous ceux de Miscou faisaient partie, mais qui n'est pas exclusive de l'existence d'une Cie particulière, car les trois préposés étaient associés avec Jean

1 Clairambault 381 , fo. 101 , à la Bibliothèque Nationale.

2 Inventaire des biens de François Fouquet, du 20 juillet 1640, minutes de Cousinet, LI, 505, aux Archives Nationales.

3 Lhermitte-Soulier, Histoire généalogique de la Noblesse de Touraine (Paris, 1665), Lm² 118, à la Bib. Nat.

4 Inventaire des biens de François Fouquet, déjà cité. 
Tuffet ( $\left.\mathrm{N}^{\circ} 38\right)$, marchand bourgeois de La Rochelle qui donna, tant pour son compte que pour le leur, un pouvoir au maître du navire Le Nicolas de Saint-Gilles ${ }^{5}$. Jean Rozée établit d'ailleurs, en 1636, un compte pour la Cie de Miscou ${ }^{6}$, dont Jean Tuffet était le préposé, lorsqu'il passa, le 23 février 1637, avec Henri Langevin, marinier du lieu de Saint-Gilles-sur-Vie, en Poitou 7, maître du navire Le Nicolas, du port de 100 tonneaux, un contrat en exécution duquel Langevin devait armer le bâtiment avec des canons pour porter directement à Miscou le commis de la Cie avec des marchandises ${ }^{8}$. Tuffet recruta, au début de $1637^{9}$, des engagés pour le compte de cette Cie de Miscou dont il fut qualifié l'un des quatre préposés pour la direction de ses affaires, le 11 avril $1637^{10}$, lorsqu'il déclara avoir exécuté les résolutions prises par Berruyer écuyer, sieur de Manselmont, Cheffault, sieur de la Renardière et Rozée, marchand de Rouen, en achetant des farines, des vins, de l'eau-de-vie et d'autres marchandises pour l'entretien des colonies, tant à Québec, par la Cie générale de la Nouvelle-France, qu'à Miscou et Archibouctou. Ce dernier lieu paraît avoir été dénommé habituellement Richibouctou et se trouver sur l'emplacement actuel de Salmon River à hauteur de l'extrémité nord de l'île Saint-Jean. Il ne doit pas être confondu avec Chedabouctou, placé plus à l'est, en face de l'Île du Cap-Breton sur l'emplacement de la future ville d'Halifax.

Les achats faits par Tuffet représentaient la cargaison dont il avait été convenu avec Langevin qui devait, lors de son arrivée à Miscou, transborder les marchandises destinées à la Grande rivière du Saint-Laurent sur un autre bâtiment, le Saint-Pierrede-Drop, dont était maître le capitaine Pierre Gringoire.

5 Registre de Teuleron de 1636, à la date, aux Archives départementales de la Charente-Maritime.

6 Continuation de la production de Nicolas Fouquet, II: 218, notre bibliothèque particulière.

7 Actuellement chef-lieu de canton de l'arrondissement des Sablesd'Olonne, département de la Vendée.

8 Liasse de Teuleron de 1637, à la date.

9 G. Debien, "Les Engagés pour le Canada au XVIIe siècle", Revue d'Histoire de l'Amérique française, VI: 221 et suiv., $8^{\circ} \mathrm{Pa} 535$, à la Bib. Nat. 10 Liasses de Cherbonnier, à la date, aux Archives départementales de la Charente-Maritime, communication de M. Delafosse, directeur. 
Langevin reconnut, le 13 avril ${ }^{11}$, avoir reçu 44 muids, 11 boisseaux de sel dont il devait décharger à Miscou la quantité ordonnée par Gaigneur, commis de la Cie, avec les lignes, plombs et autres ustensiles destinés à la pêche. Il lui faudrait ensuite rapporter les marchandises que Gaigneur lui remettrait.

Les personnalités des associés étaient susceptibles de modifications, car François Fouquet céda, le 10 décembre 1638, une de ses parts pour 1,000 livres, le prix qu'elle lui avait coûté, au fameux marin havrais, noble homme Jacob Bontemps ${ }^{12}$, qualifié capitaine ordinaire de la marine, alors demeurant à Dieppe. Bontemps lui acheta le même jour un des deux quarante-cinquièmes de part de la société des Iles Saint-Christophe et adjacentes provenant du président Cornuel, suivant acte du Ier février 1635 passé chez le même notaire, l'autre ayant été cédé à Pierre Chanut, trésorier de France en Auvergne, associé comme Bontemps, $\left(\mathrm{N}^{\circ} 126\right)$ de la Cie de la Nouvelle-France ${ }^{13}$, sous le $\mathrm{N}^{\circ} 145$.

Les préposés de la Cie de Miscou étaient également remplaçables, car, le 9 avril 1641, Cheffault agissait pour noble homme Pierre Robineau, conseiller du roi, trésorier général de la cavalerie légère, figurant au $\mathrm{N}^{\circ} 42$ de la liste des Cent-Associés et Jean Verdier, secrétaire du roi, figurant au $\mathrm{N}^{\circ} 59$, lorsqu'il passa une charte-partie avec Vincent Achard, demeurant à Saint-Gillessur-Vie en Poitou, maître après Dieu du navire La Charlotte, de 75 à 80 tonneaux, armé de 4 canons, pour transporter des marchandises et employer le sel à lui confié pour faire une pêche de morues vertes aussi importante que possible ${ }^{14}$. L'activité de Cheffault continuait d'être double, car il passa, le même jour et devant le même notaire, une autre charte-partie pour le compte de la Cie du Saint-Laurent, à destination de Tadoussac. Il en passa encore une autre, le sept mai 1641, comme directeur de la cie de la Nouvelle-France, avec Nicolas Pernet, des Sables d'Olonne, maître après Dieu du navire Le René, de 120 tonneaux, en partance de La Rochelle, se portant fort pour Robineau, Ver-

\footnotetext{
11 Registre de Teuleron de 1637, fo. 36.

12 Cousinet, 51,500 .

13 Idem.

14 Registre de Teuleron, 1641, fo. 51, vo.
} 
dier, les marchands de Rouen Louis Mousnier $\left(\mathrm{N}^{\circ} 146\right)$, et Jean Rozée, tous préposés aux embarquements de la Cie particulière pour le fleuve Saint-Laurent, Tadoussac et Québec ${ }^{15}$.

Cheffault engagea à La Rochelle, du 2 au 16 avril 1642, du personnel pour Miscou ${ }^{16}$. Il en fit autant, avec Nicolas Denys, agissant, comme lui pour le compte de la Cie de La NouvelleFrance, les 30 mars et 14 avril $1643^{17}$, mais des pourparlers pour la cession de Miscou furent engagés avec Nicolas Denys avant le 27 février 1645, date à laquelle ce dernier donna une procuration devant le notaire rochelais Teuleron ${ }^{18}$ à son frère Jacques, écuyer, capitaine d'un des navires de l'armée navale du roi, pour emprunter à la grosse aventure ou autrement et prendre à rente des associés de la Cie de Miscou l'habitation de ce nom avec le pouvoir de traiter en Nouvelle-France à eux concédé par la Cie des Cent-Associés.

Cette habitation devait se trouver si l'on en croit ce qu'écrivit plus tard Nicolas Denys dans une île portant le même nom au sud de l'entrée de la baie des Chaleurs, au-delà du passage de Caraqui, à dix lieues en mer ${ }^{19}$. Les pêcheurs basques continuaient de fréquenter la région, car Thierry Desdames, gouverneur sur place pour la Cie de Miscou et le père Léon, jésuite résidant en ce lieu, s'étaient vus confier par le capitaine Joannès de Béreau dix chaloupes que Joannot de Haraneder et Jean de Latrie, bourgeois et marchands de Ciboure, vendirent, le 17 mars 1645, pour 43 livres pièce à Auger Duchanin, marchand bourgeois de La Rochelle et à Jacques Burique, chargé d'affaires d'honorable homme Jean Darrasson, bourgeois et marchand d'Hennebont, en Bretagne ${ }^{20}$. Thierry Desdames, était un marchand de Rouen où il demeurait quand il avait donné, dès le 3 janvier 1634, une procuration à Me Jacques de Mounès, avocat, aux fins de faire restituer par Pierre de Nesfle, capitaine de

15 I dem, fo. 62 , vo.

16 Idem, registre de 1642, fo. 49 et G. Debien, op. cit., 225.

17 Idem, 227 et 228.

18 Minutes de Nicolas Motelet, notaire au Châtelet, XC: 208. 499 .

19 Ganong, Description and Natural History of Acadia (1908), 498 et

20 Registre de Teuleron, 1645 , fo. 4 , vo. 
navire demeurant à Dieppe, deux robes de castor qui lui avaient été remises à Québec, par un certain Jean Pointel ${ }^{21}$.

La cession au profit de Nicolas Denys et de son frère Jacques ayant élu domicile chez un certain Harlan, marchand demeurant rue Saint-Denis, à Paris, fut réalisée pour neuf ans, le 25 avril 1645 suivant acte passé devant Nicolas Motelet, notaire au Châtelet, par Jacques et Georges Berruyer, ce dernier demeurant rue des Sept Voies, près du collège de Montaigne, Verdier, Robineau et Cheffault, associés que nous connaissons. Ils étaient renforcés par messire Jacques de La Ferté, prêtre, abbé de Sainte-Madeleine, chantre et chanoine de l'église de la SainteChapelle à Paris, demeurant en l'enclos du Palais, associé de la Cie de la Nouvelle-France sous le $\mathrm{N}^{\circ} 97$, par messire Jacques Bordier, conseiller du roi en ses Conseils, secrétaire du Conseil d'État, demeurant rue du Parc du Roi, associé sous le $\mathrm{N}^{\circ} \mathbf{5 5}$, par Pierre Desportes, écuyer, sieur de Lignères et du Cap-Breton, associé sous le $\mathrm{N}^{\circ} 79$, par noble homme Claude Margonne, secrétaire du roi, receveur général des finances à Soissons, demeurant rue du Parc du Roi, associé sous le $\mathrm{N}^{\circ} 56$, par noble homme Martin Tabouret, aussi secrétaire du roi, demeurant rue Vieille du Temple $\left(\mathrm{N}^{\circ} 146\right)$ par André Céberet, écuyer sieur du Grand Boullé, conseiller du roi, commissaire général de la Marine, demeurant rue Baillette, paroisse Saint-Germain-l'Auxerrois $\left(\mathrm{N}^{\circ}\right.$ 100), par Claude de Bragelonne, écuyer, sieur de Boisripault, demeurant rue Saint-Antoine $\left(\mathrm{N}^{\circ} 54\right)$. Tous associés de la Cie de Miscou, ils se portaient forts pour d'autres qui étaient absents.

Moyennant le paiement annuel de 200 peaux d'orignaux, bonnes, loyales et marchandes livrées à La Rochelle à Robineau et Margonne, alors directeurs de la Cie, la jouissance pour les frères Denys des droits que la Cie de Miscou tenait de celle des Cent-Associés devait commencer, soit à partir de l'arrivée de leur navire à Miscou, soit à partir de la connaissance du contrat par Thierry Desdames ou tout autre commandant en sa place à l'habitation que l'acte situait en la baie des Chaleurs. Les Denys étaient tenus de l'entretenir et de la restituer dans l'état où

21 Tabellionage de Rouen, meubles, janvier-février 1634, à la date, aux Archives de la Seine-Maritime. 
ils la prendraient constaté par procès-verbal. Ils pouvaient y nommer un capitaine ou un commis de leur choix pourvu par la Cie, mais il leur fallait payer 1,200 livres de pension aux pères jésuites qui s'y trouvaient installés, même s'ils changeaient leur lieu d'habitation, passer sans indemnité les vivres et autres choses nécessaires à ces religieux, le risque de la mer restant cependant à la charge de ces derniers. Sous la réserve d'obtenir une concession de la Cie de Miscou, ils pouvaient défricher des terres et établir des habitations qui resteraient leur propriété en fin de contrat, lorsqu'ils rendraient celle de Miscou.

L'étendue de leur privilège pour la traite allait depuis Canso jusqu'à Gaspé ou le cap des Rosiers, et aussi loin qu'ils pourraient pénétrer dans l'intérieur des terres. Ce territoire était interdit, tant à d'Aulnay, établi au Port-Royal, qu'à Guignard, installé au Cap-Breton. Par réciprocité, les Denys devaient s'abstenir de traiter sur des terres concédées à d'autres. Il leur était imposé de retirer des mains des sauvages les pelleteries dues par eux à la Cie et d'apporter à La Rochelle toutes les pelleteries traitées pour son compte avant leur entrée en possession, de rembourser la valeur des marchandises se trouvant à Miscou estimée d'après les factures avec une augmentation de $20 \%$ représentant les frais et risques du transport en NouvelleFrance, d'acheter les armes, les munitions et le matériel de la Cie au prix fixé contradictoirement entre, d'une part, Thierry Desdames, Prévost et Legay, commis de la Cie, d'autre part, trois personnes désignées par les acquéreurs, avec arbitrage éventuel du révérend père supérieur des Jésuites, les paiements devant être effectués moitié au retour du navire des Denys en 1645, moitié au retour de 1646. En fin de contrat, la Cie devait reprendre les pelleteries, armes, munitions et matériel aux mêmes conditions ${ }^{22}$.

Dès le 5 mai 1645 , Nicolas Denys, propriétaire et avitailleur du navire rochelais de 300 tonneaux le Saint-Joseph, lui institua un maître en la personne de Jacques Pacquier. Avec $\mathbf{7 5}$ hommes d'équipage, 21 canons et 40 mousquets, il devait transporter des

22 XC, 208, aux Arch. Nationales. 
marchandises et les remettre au gouverneur de Miscou avant de faire la pêche, moyennant une indemnité constituée par toutes les "meugres" et langues de morues. Il semble qu'il y eut une collaboration tout au moins provisoire avec la Cie de Miscou, car Pacquier devait, pendant le voyage reconnaître pour capitaine un certain Prévost ${ }^{23}$. Ce personnage pouvait être Pierre Prévost qui recruta à La Rochelle, du 2 au 16 avril 1642, puis, du 10 avril au 2 mai 1644, du personnel pour le compte de la Cie des Cent-Associés, et d'un sieur de Chauvigny, écuyer, résidant en Nouvelle-France ${ }^{24}$, mais il s'agissait, plus vraisemblablement, de Gabriel Prévost, commis de la Cie de Miscou, qui faisait, avant le 2 septembre 1645, sur l'ordre de Thierry Desdames, commandant le fort de Miscou, le troc à Richibouctou lorsque Bernard Marot, commandant pour d'Aulnay deux chaloupes du navire Le Cardinal l'obligea de s'y embarquer pour être conduit au Port-Royal où Charles de Menou lui confisqua ses marchandises ${ }^{25}$.

Sauvic, capitaine du Grand-Cardinal, exposa dans un rapport du 8 novembre 1645, qu'il avait capturé un navire flamand, Le Lion Rouge, d'Amsterdam, une barque dépendant de l'habitation de Tuffet au Cap-Breton et une barque appartenant à la Cie de Miscou ${ }^{26}$. La déposition du capitaine hollandais en date du 6 novembre confirme qu'il avait été fait prisonnier à Richibouctou, dans les dépendances de la Cie de Miscou ${ }^{27}$ et, le 7 novembre 1645, un sieur de Corbye, agissant pour la Cie de la NouvelleFrance, se plaignit de Sauvic, Marot et Habrard qu'il accusait de violences et malversations ${ }^{28}$. Celles-ci ne paraissaient pas porter sur 500 peaux d'orignaux à 30 livres pièce, 33 peaux de castors et 15 peaux de loutres à 10 livres chacune que Sauvic vendit à Emmanuel Leborgne pour 15480 livres tournois comme appartenant à lui et à son équipage parce qu'elles représentaient le

23 Registre de Teuleron de 1645 , fo. 57.

24 G. Debien, "Engagés pour le Canada", op. cit., 224. Maritime.

25 Rapport de Gabriel Prévost, B 5656 aux Arch. Dép. de la Charente

26 Rapport de Sauvic, Idem.

27 B 5657, Idem.

28 Rapport de Prévost, déjà cité. 
paiement de leur fret par d'Aulnay ou le résultat d'opérations de troc suivant acte du 19 octobre $1645^{29}$.

Cette courte étude de la Cie de Miscou nous permet de penser qu'il est difficile pour le début du XVIIème siècle de distinguer l'histoire de l'Acadie de celle de la région du SaintLaurent. Elle nous introduit dans le milieu pittoresque des Associés de la Cie de la Nouvelle-France, nous confirmant que Jacques Bordier et Claude Margonne étaient bien voisins comme le signale Tallemant des Réaux qui sut leur trouver un autre point commun ${ }^{30}$.

ROBERT LE BLANT

33 , rue de Turin, Paris $8^{\circ}$. 